\title{
Genetic diversity of transmission-blocking vaccine candidates Pvs25 and Pvs28 in Plasmodium vivax isolates from Yunnan Province, China
}

\author{
Hui Feng ${ }^{1,2}$, Li Zheng ${ }^{1}$, Xiaotong Zhu' ${ }^{1}$, Gege Wang ${ }^{1}$, Yanyan Pan ${ }^{1}$, Ying Li ${ }^{1}$, Yimei Yang ${ }^{3}$, Yahui Lin ${ }^{4}$ Liwang Cui ${ }^{5}$ \\ and Yaming $\mathrm{CaO}^{1 *}$
}

\begin{abstract}
Background: Transmission-blocking vaccines (TBVs) have been considered an important strategy for disrupting the malaria transmission cycle, especially for Plasmodium vivax malaria, which undergoes gametocytogenesis earlier during infection. Pvs25 and Pvs28 are transmission-blocking vaccine candidates for $P$. vivax malaria. Assessment of genetic diversity of the vaccine candidates will provide necessary information for predicting the performance of vaccines, which will guide us during the development of malaria vaccines.

Results: We sequenced the coding regions of pvs 25 and pvs 28 from 30 P. vivax isolates from Yunnan Province, identifying five amino acid haplotypes of Pvs 25 and seven amino acid haplotypes of Pvs28. Among a total of four mutant residues, the predominant haplotype of Pvs25 only had the 1130T substitution. For Pvs28, a total of eight amino acid substitutions were identified. The predominant haplotype of Pvs28 had two substitution at positions 52 (M52L) and 140 (T140S) with 5-6 GSGGE/D tandem repeats at the end of fourth EGF-like domain. Most amino acid substitutions were common with previous reports from South Asian isolates. Although the nucleotide diversity of pvs28 ( $\pi=0.0034 \pm 0.0012)$ was significantly higher than pvs25 $(\pi=0.0013 \pm 0.0009)$, it was still conserved when compared with the blood stage vaccine candidates.
\end{abstract}

Conclusions: Genetic analysis revealed limited genetic diversity of pvs 25 and pvs28, suggesting antigenic diversity may not be a particular problem for Sal I based TBVs in most P. vivax-endemic areas of China.

Keywords: Plasmodium vivax, TBVs, Pvs25, Pvs28, polymorphism

\section{Background}

Plasmodium vivax, the most widespread species of human malaria parasites, is responsible for the majority of malaria cases outside of Africa, and the most prevalent form of relapsing malaria. Although not as lethal as P. falciparum malaria, $P$. vivax has caused substantial morbidity for human populations residing in Asia and South America [1,2]. Throughout the malaria control history, $P$. vivax malaria has displayed tremendous resilience to control efforts, which is in part due to two biological characteristics of this parasite. One is the formation of hypnozoites in the liver, which is responsible for relapses

\footnotetext{
* Correspondence: ymcao@mail.cmu.edu.cn

'Department of Immunology, College of Basic Medical Sciences, China

Medical University, Shenyang, China Full list of author information is available at the end of the article
}

of the disease. The other is that $P$. vivax undergoes gametocytogenesis before manifestation of the disease symptoms, making transmission possible before treatment.

P. vivax is the predominant Plasmodium species in China, and in recent years, $P$. vivax cases accounted for more than $90 \%$ of all malaria cases [3]. As The Ministry of Health of China has set the goal of malaria elimination by 2020 [4], interruption of vivax malaria transmission is a major challenge. Yunnan is one of the two provinces that have year-round local transmission of $P$. vivax and P. falciparum. It is located in southwest China and borders Myanmar to the west and Laos and Vietnam to the south [5]. A total of 250,070 confirmed cases of $P$. vivax malaria and 44,465 cases of $P$. falciparum malaria have been reported between 1991 and 2006 in Yunnan [6]. In recent years, we have witnessed a significant decline of
C Biomed Central 
malaria cases in Yunnan [3]. A total of 32,566 confirmed cases of $P$. vivax malaria and 5,821 cases of $P$. falciparum malaria have been reported between 2006 and 2009 in Yunnan [3,7-9]. Most of the malaria cases are now clustered in counties located at the China-Myanmar border area $[3,10]$. Despite improvement in malaria situation in this region, malaria control along the borders is problematic, since reintroduction of malaria by human migration is still difficult to monitor. Thus, it is particularly important to develop effective strategies to control and eliminate malaria in this region.

Transmission-blocking vaccines (TBVs), which target the sexual stages of malaria parasites to prevent their further development within mosquitoes, are considered an important strategy for disrupting malaria transmission $[11,12]$. To date, several sexual stage antigens have been characterized, which showed excellent transmission blocking activities. These include the pre-fertilization antigens P48/45 and post-fertilization antigens P25 and P28. P25 and P28, are specifically expressed on Plasmodium zygote and ookinete surface [11], and they are essential for the survival of ookinetes in the mosquito midgut, and subsequent penetration of the midgut epithelium and transformation into oocysts [13]. Among the TBVs candidates, Pvs25 has received the most attention. Recombinant Pvs25 protein expressed in bacteria [14], yeasts [15,16] and baculovirus [17] exhibits strong immunogenicity and immune sera display significant transmission-blocking activity on the development of sporozoites [14] and oocysts [15-17]. The immunogenicity trials of recombinant Pvs25 have been undertaken in rhesus monkeys [18] and human volunteers $[19,20]$. A phase I clinical trial of Pvs25H vaccine in human volunteers has demonstrated that vaccineinduced antibodies have significant transmission-blocking activity [19]. It has been reported that antisera to recombinant Pvs 25 and Pvs28 based on the Sal-I strain recognized the corresponding molecules expressed by field-isolated parasites in Thailand, and that these antisera blocked transmission of field isolates [15].

Polymorphisms represent a major impediment in vaccine design. An effective vaccine must either be based on conserved regions or incorporate multiple allelic forms of the antigen. As antigenic polymorphisms limit the immunogenicity and immunoreactivity of vaccines, it is necessary to understand antigenic variations in order to generate effective vaccines against natural Plasmodium infections [12]. Pvs25 and Pvs28 contained conserved structures, which are characterized by a secretory $\mathrm{N}$ terminal signal sequence followed by four epidermal growth factor (EGF) domains and a glycosylphosphatidylinositol (GPI) anchor [21]. To date, genetic diversity of pvs 25 and pvs 28 genes has been surveyed in several Asian countries, including South Korea [22], Indonesia [23], Iran [24], India [25,26], Bangladesh [25], and
Thailand [15]. In this study, we aim to analyze the genetic diversity of these candidate TBVs genes in parasite isolates from Yunnan Province, China.

\section{Methods}

\section{Study areas}

Yunnan Province is one of the most highly endemic regions in China with year-round transmission of P. vivax and P. falciparum. Since 2001, there has been a strong seasonal pattern of malaria incidence characterized by a peak of infection occurring from June to July and another peak from October to November [27]. Counties with higher malaria incidence rates are clustered in the border area and the Yuanjiang River Basin [6,27]. The predominant malaria vectors are Anopheles minimus and Anopheles dirus [28].

\section{Parasite collection}

A total of 30 parasite samples were collected in 2004 from three regions in Yunnan. All volunteers in this study were symptomatic patients diagnosed with $P$. vivax malaria using Giemsa stained thin smear examination by malaria clinic staff. After informed consent/ assent was obtained from either adults or parents or legal guardians of children, $\sim 100 \mu \mathrm{l}$ of finger-prick blood were collected from each patient on filter papers. The patients were Yunnan local residents, 13 to 50 years old, living in Baoshan (10 individuals), Ruili (10 individuals) and Longchuan (10 individuals) (showed in Figure 1). Patients were then treated according to the Ministry of Health drug policy. This study protocol was approved by the Biomedical Research Ethics Review Board at China Medical University.

\section{Parasite DNA preparation and amplification}

Three circles, $2.5 \mathrm{~mm}$ in diameter, were punched out from each dried blood spot and Plasmodium DNA was purified using the QIAamp DNA Mini kit (QIAGEN, Hilden, Germany) according to the manufacturer's protocol. DNA was eluted in $60 \mu \mathrm{l}$ of $\mathrm{H}_{2} \mathrm{O}$. pvs25 (GenBank: AF083502.1) and pvs28 (GenBank: AF083503.2) of the Salvador I (Sal I) strain were used as reference sequences. The full-length open reading frames (ORFs) of $p v s 25$ and $p v s 28$ were amplified using the following primer pairs: pvs25F (5'CACTTAGCCAAAATGAACTC-3') and pvs25 R (5'AAAGGACAAGCAGGATGATA-3') for $p v s 25$; $p v s 28 \mathrm{~F}$ (5'-CTACCACAGCTTGCTGTTCC-3') and pvs28R (5'TGACATCATGAAGAAGGCG-3') for $p v s 28$. The PCR reaction contained $2 \mu \mathrm{l}$ of $10 \times$ KOD-Plus buffer, $2 \mu \mathrm{l}$ of $2 \mathrm{mM}$ dNTPs, $0.8 \mu \mathrm{l}$ of $25 \mathrm{mM} \mathrm{MgSO}_{4}, 1.0 \mu \mathrm{l}$ of $10 \mu \mathrm{M}$ of each primer, 0.2 units of KOD-Plus DNA polymerase (Toyobo, Osaka, Japan), and $0.5 \mu$ genomic DNA template in a final volume of $20 \mu \mathrm{l}$. Amplification conditions were as follows: initial denaturing at $94^{\circ} \mathrm{C}$ for $2 \mathrm{~min}, 35$ cycles 


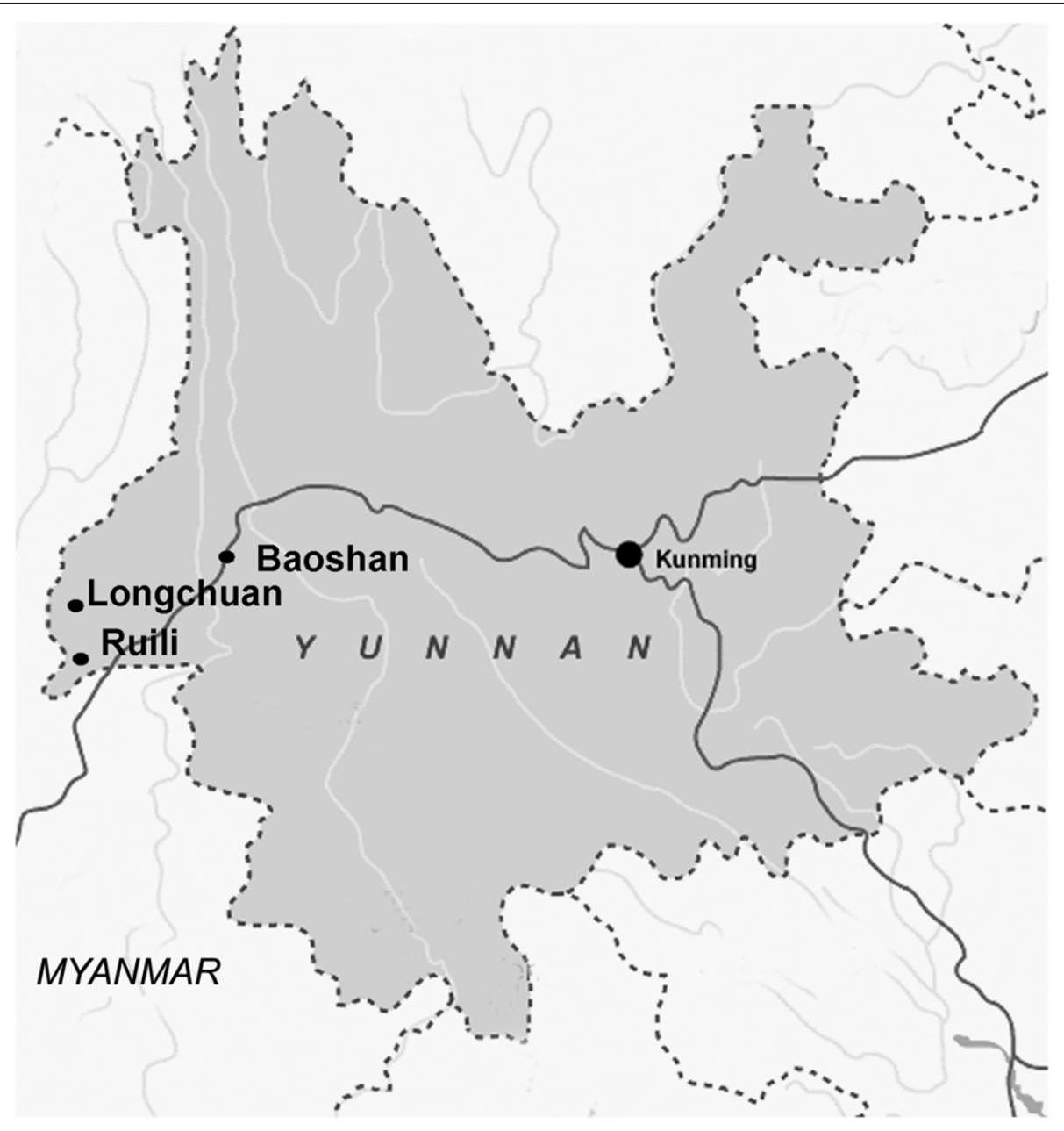

Figure 1 Map of the Yunnan Province of China to show the sampling sites.

of $94^{\circ} \mathrm{C}$ for $15 \mathrm{sec}, 56^{\circ} \mathrm{C}$ (for pvs 28 ) or $54^{\circ} \mathrm{C}$ (for $p v s 25$ ) for $15 \mathrm{sec}$, and $68^{\circ} \mathrm{C}$ for $1 \mathrm{~min}$, and a final extension at $68^{\circ} \mathrm{C}$ for 5 min. PCR products were cleaned up using Exonuclease I and Shrip alkaline phosphatase treatment according to the manufacturer's instructions (USB, CA, USA).

\section{DNA sequencing}

Pvs 25 and pvs 28 ORFs were sequenced using the ABI Prism $^{\circledR}$ BigDye $^{\mathrm{TM}}$ Terminator cycle sequencing kit (Applied Biosystems, Foster City, CA, USA) on an ABI PRISM $^{\circledR} 310$ Genetic Analyzer (Applied Biosystems, Foster City, CA, USA). Sequencing primers are: pvs25F (5'GAAACCCTAGGCAAAGCATG-3') and $p v s 25 \mathrm{R}$ (5'- GG GACTTTGCCA ATAGCACA-3') for $p v s 25$, and $p v s 28 \mathrm{~F}$ (5'-AACTGTGGAGACTACGCTG-3') and pvs28R (5'ATATTACAAGAGCACATGGTG-3') for $p v s 28$.

\section{Statistical Analysis}

The ORFs of $p v s 25$ and $p v s 28$ were aligned with the reference sequences using the CLUSTAL W program
[29] with manual corrections. Nucleotide diversity $(\pi)$ and its standard error (SE) were calculated with the Jukes and Cantor method using MEGA 4.0. The number of haplotypes $(h)$, haplotypes diversity $(H d)$, segregating sites $(S)$ were computed using the options available in DnaSP 5.0 [30].

\section{Results}

Sequence polymorphisms of the pvs 25 and pvs 28 genes Pvs 25 and pvs 28 genes were successfully amplified in 30 $P$. vivax samples obtained from Yunnan. The sequence of pvs25 (645 bp) and pvs28 (712 bp) were determined by direct sequencing of the PCR products. The $30 \mathrm{pvs} 25$ sequences contained four polymorphic nucleotide sites (C103A, G289C, T389C, and C391A), which resulted in four amino acid substitutions (L35M, E97Q, I130T, and Q131K). These substitutions resulted in a total of five amino acid haplotypes, hitherto referred to as Pvs25-I, II, III, IV and V (Table 1). The predominant amino acid haplotype of Pvs25 only had the I130T substitution. 
Table 1 Amino acid substitutions of Pvs25 from Yunnan $P$. vivax isolates

\begin{tabular}{ccccc}
\hline Haplotypes (number) & EGF-1 & EGF-2 & EGF-3 & EGF-3 \\
& $\mathbf{3 5}$ & $\mathbf{9 7}$ & $\mathbf{1 3 0}$ & $\mathbf{1 3 1}$ \\
\hline Sal-I & L & E & I & Q \\
Pvs25-I $(n=15)$ & $\cdot$ & $\cdot$ & $T$ & $\cdot$ \\
Pvs25-II $(n=8)$ & $\cdot$ & $\cdot$ & $T$ & K \\
Pvs25-III $(n=5)$ & $\cdot$ & Q & $T$ & $\cdot$ \\
Pvs25-IV $(n=1)$ & $\cdot$ & Q & T & K \\
Pvs25-V $(n=1)$ & M & $\cdot$ & $T$ & $K$ \\
\hline
\end{tabular}

EGF, epidermal growth factor-like domain. • indicates identical amino acid residues compared to the Salvador I strain.

The 30 pvs 28 sequences had nucleotide polymorphisms at 13 sites, which included four synonymous substitutions (T346C, T558C, C573T, and G579A) and eight nonsynonymous substitutions (G40T, A154C, T292A, G313A, T346G, C419G, G536A, and C666G), which resulted in eight amino acid substitutions (V14L, M52L, L98I, E105K, L116V, T140S, G179E, and I222M). A total of seven amino acid haplotypes were identified: Pvs28-I, II, III, IV, V, VI and VII (Table 2). The predominant amino acid haplotype of Pvs28 had two amino acid substitutions at positions 52 (M52L) and 140 (T140S). Compared with Sal-I strain, we found the majority of clones had six copies of GSGGE/D tandem repeats at the end of fourth EGFlike domain. Only one clone with Pvs28-I haplotype had five copies of repeat regions.

\section{Compared with the worldwide isolates}

Compared with previous reports of worldwide isolates, Pvs25-I haplotype showed $100 \%$ identity with isolates from Vietnam (GenBank: ABG29072.1), North Korea (GenBank: AAV33640.1) [23], Thailand (GenBank: AB091730.1) [15] and Mexico (GenBank: ABS70906.1ABS70935.1) [31]. The Pvs25-II haplotype was similar to some isolates from Bangladesh (GenBank: BAA94348.1BAA94350.1) [25] and Thailand (GenBank: BAC66001.1) [15]. The Pvs25-III haplotype was common with isolates from Iran (GenBank: ACJ54129.1, ACJ54128.1) [24], Thailand (GenBank: BAC66003.1) [15], Indonesia (GenBank: AAV33639.1) [23], India (GenBank: BAA94346.1) [25] and Bangladesh (GenBank: BAA94347.1) [25]. Only Pvs25-IV was unique in China. Like in some South Korean isolates, position 35 was also polymorphic, but the substitution is L35M in the Yunnan isolate as compared to L35P in South Korean isolates (GenBank: ADJ18839.1) [22].

The Pvs28-I haplotype in Yunnan isolates was also common in isolates from Iran (GenBank: ACJ54136.1) [24] and Bangladesh (GenBank: BAA94352.1, BAA94353.1, BAA94371.1, BAA94362, BAA94363) [25]. The Pvs28II haplotype was similar with those from Thailand (GenBank: BAC66011.1) [15] and Bangladesh (GenBank: BAA94370.1, BAA94369.1) [25]. Parasite isolates from Yunnan contained some region-specific haplotypes. Haplotype Pvs28-III with a new V14L substitution was found only in one isolate. The remaining haplotypes (Pvs28-IV, $\mathrm{V}$ and VI) were only identified in the Yunnan isolates. The G179E mutation was only found in South Korean (GenBank: ADJ18881.1) [22] and Chinese isolates.

The Pvs25 and Pvs28 amino acid substitutions identified from worldwide isolates including those from China are summarized in Tables 3 and 4. Among a total of 17 variant amino acids in Pvs25 from worldwide isolates, I130T was the most common amino acid substitution from American and Asian isolates. The Q87K substitution was common in American isolates such as those from Brazil [23] and Mexico [31], and Western Asian isolates such as those from Iran [24] and Turkey, but not detected in South and East Asian isolates. Amino acid residue E97Q was detected in some Asian isolates such as those from Iran [24], Bangladesh [25], Thailand [15], India [25,26], Indonesia [23], South Korea [22], Central China [32] and Yunnan, but rare in American isolates. Compared with previous studies from Central China isolates, amino acid residue $\mathrm{I} 130 \mathrm{~T}$ was the major amino acid substitution in Yunnan, Zhejiang and Hubei isolates [32]. The amino acid residue Q131K was not detected in

Table 2 Amino acid substitutions of Pvs28 from Yunnan $P$. vivax isolates

\begin{tabular}{|c|c|c|c|c|c|c|c|c|c|}
\hline \multirow[t]{2}{*}{ Haplotypes (number) } & SS & EGF-1 & EGF-2 & EGF-2 & EGF-3 & EGF-3 & RR & RR & THR \\
\hline & 14 & 52 & 98 & 105 & 116 & 140 & 179 & $\#$ & 222 \\
\hline Sal-I & V & M & L & $E$ & L & $\mathrm{T}$ & G & 6 & I \\
\hline Pvs28-I $(n=19)$ & · & $\mathrm{L}$ & . & . & . & $\mathrm{S}$ & . & $5 / 6$ & \\
\hline Pvs28-II (n = 4) & $\cdot$ & - & I & - & V & - & - & 6 & $\cdot$ \\
\hline Pvs28-III (n = 2) & L & · & · & K & V & · & · & 6 & \\
\hline Pvs28-IV ( $n=2)$ & $\cdot$ & $\mathrm{L}$ & • & · & - & • & • & 6 & 。 \\
\hline Pvs28-V $(n=1)$ & $\cdot$ & · & • & · & · & · & - & 6 & M \\
\hline Pvs28-VI $(n=1)$ & $\cdot$ & · & • & · & V & · & · & 6 & M \\
\hline Pvs28-VII $(n=1)$ & - & $\mathrm{L}$ & - & - & - & - & $E$ & 6 & \\
\hline
\end{tabular}

SS, secretary signal sequence; EGF, epidermal growth factor-like domain; RR, repeat regions, \# indicates the number of the GSGGE/D repeats; THR, the C terminal hydrophobic region.

- indicates identical amino acid residues compared to the Salvador I strain. 
Table 3 Amino acid variations of Pvs25 among worldwide isolates

\begin{tabular}{|c|c|c|c|c|c|c|c|c|c|c|c|c|c|c|c|c|c|c|}
\hline \multirow[b]{2}{*}{ Country } & \multirow{2}{*}{$\begin{array}{c}\text { SS } \\
2\end{array}$} & \multicolumn{2}{|c|}{ EGF-1 } & \multicolumn{2}{|c|}{ EGF-2 } & \multicolumn{6}{|c|}{ EGF-3 } & \multicolumn{5}{|c|}{ EGF-4 } & \multirow{2}{*}{$\frac{\text { THR }}{199}$} & \multirow[b]{2}{*}{ Ref. } \\
\hline & & 35 & 38 & 87 & 97 & 130 & 131 & 132 & 137 & 138 & 149 & 170 & 174 & 183 & 196 & 198 & & \\
\hline Sal-I & $N$ & L & M & $\mathrm{Q}$ & $E$ & 1 & Q & $S$ & C & A & K & $C$ & $E$ & $E$ & $S$ & $S$ & V & AAC9976 \\
\hline Iran & $\cdot$ & . & $\cdot$ & $\mathrm{Q} / \mathrm{K}$ & $E / Q$ & $\mathrm{~T}$ & $\cdot$ & . & - & - & . & . & . & . & - & . & . & [24] \\
\hline Mexico & - & - & - & $\mathrm{Q} / \mathrm{K}$ & $\cdot$ & $\mathrm{I} / \mathrm{T}$ & - & - & . & - & - & . & . & - & - & . & . & [31] \\
\hline Brazil & - & - & - & K & . & $\cdot$ & $\cdot$ & - & . & - & - & - & . & . & . & . & - & [23] \\
\hline Colombia & $\cdot$ & $\cdot$ & $\cdot$ & K & $\cdot$ & $\cdot$ & $\cdot$ & $\cdot$ & $\cdot$ & $\cdot$ & • & $\cdot$ & $\cdot$ & $\cdot$ & $\cdot$ & $\cdot$ & $\cdot$ & [23] \\
\hline Venezuela & $\cdot$ & $\cdot$ & $\mathrm{T}$ & K & - & $\cdot$ & $\cdot$ & $\cdot$ & - & • & - & - & . & $\cdot$ & $\cdot$ & . & • & [23] \\
\hline Mauritania & $\cdot$ & $\cdot$ & · & K & • & $\mathrm{T}$ & $\cdot$ & . & - & $\cdot$ & - & . & . & • & • & . & • & [23] \\
\hline Turkey & $\cdot$ & $\cdot$ & $\cdot$ & K & · & $\mathrm{T}$ & $\cdot$ & $\cdot$ & · & $\cdot$ & $\cdot$ & · & · & • & · & · & $\cdot$ & ABG29073 \\
\hline PNG & $\cdot$ & $\cdot$ & $\cdot$ & $\cdot$ & $\cdot$ & $\mathrm{T}$ & K & R & $\cdot$ & $\cdot$ & $N$ & $\cdot$ & $\cdot$ & $\cdot$ & $\cdot$ & $\cdot$ & $\cdot$ & [23] \\
\hline Nicaragua & $\cdot$ & $\cdot$ & $\cdot$ & • & $\cdot$ & $\cdot$ & $\cdot$ & $\cdot$ & $\cdot$ & $\cdot$ & $\cdot$ & $\mathrm{R}$ & $\cdot$ & $\cdot$ & $\cdot$ & $\cdot$ & $\cdot$ & [23] \\
\hline India & $\cdot$ & $\cdot$ & $\cdot$ & - & Q & $\mathrm{T}$ & $\cdot$ & $\cdot$ & $\cdot$ & $\cdot$ & • & · & $\cdot$ & $\cdot$ & $\cdot$ & $\cdot$ & $\cdot$ & {$[25]$} \\
\hline India & $\cdot$ & $\cdot$ & $\cdot$ & $\cdot$ & $E / Q$ & $\mathrm{~T}$ & $\mathrm{Q} / \mathrm{K}$ & $\cdot$ & CN & $\mathrm{A} / \mathrm{G}$ & - & $\cdot$ & $E / K$ & $E / K$ & $S / F$ & $S / T$ & $\mathrm{~V} / \mathrm{E}$ & {$[26]$} \\
\hline Bangladesh & $\cdot$ & $\cdot$ & $\cdot$ & · & $E / Q$ & $\mathrm{~T}$ & $\mathrm{Q} / \mathrm{K}$ & · & $\cdot$ & $\cdot$ & • & $\cdot$ & $\cdot$ & $\cdot$ & $\cdot$ & $\cdot$ & $\cdot$ & {$[25]$} \\
\hline Vietnam & $\cdot$ & • & $\cdot$ & $\cdot$ & $\cdot$ & $\mathrm{T}$ & $\cdot$ & $\cdot$ & $\cdot$ & • & · & $\cdot$ & $\cdot$ & $\cdot$ & $\cdot$ & $\cdot$ & $\cdot$ & ABG29072 \\
\hline Indonesia & $\cdot$ & $\cdot$ & $\cdot$ & $\cdot$ & Q & $\mathrm{T}$ & $\cdot$ & $\cdot$ & $\cdot$ & $\cdot$ & $\cdot$ & $\cdot$ & $\cdot$ & $\cdot$ & $\cdot$ & $\cdot$ & $\cdot$ & [23] \\
\hline Thailand & $\cdot$ & $\cdot$ & $\cdot$ & - & $E / Q$ & $\mathrm{~T}$ & $\mathrm{Q} / \mathrm{K}$ & • & $\cdot$ & $\cdot$ & • & $\cdot$ & $\cdot$ & $\cdot$ & $\cdot$ & $\cdot$ & $\cdot$ & {$[15]$} \\
\hline N Korea & $\cdot$ & $\cdot$ & $\cdot$ & $\cdot$ & $\cdot$ & $\mathrm{T}$ & $\cdot$ & $\cdot$ & $\cdot$ & $\cdot$ & • & $\cdot$ & $\cdot$ & $\cdot$ & $\cdot$ & $\cdot$ & $\cdot$ & {$[23]$} \\
\hline S Korea & $\mathrm{N} / \mathrm{D}$ & $\cdot$ & - & - & $E / Q$ & $\mathrm{~T}$ & . & - & . & - & - & - & . & - & - & . & - & {$[22]$} \\
\hline China (ZJ/HB) & $\cdot$ & $\cdot$ & $\cdot$ & $\cdot$ & $E / Q$ & $\mathrm{~T}$ & $\cdot$ & $\cdot$ & $\cdot$ & $\cdot$ & $\cdot$ & $\cdot$ & $\cdot$ & $\cdot$ & $\cdot$ & $\cdot$ & $\cdot$ & [32] \\
\hline China (YN) & - & $L / M$ & - & $\cdot$ & $E / Q$ & $\mathrm{~T}$ & $\mathrm{Q} / \mathrm{K}$ & $\cdot$ & - & - & - & - & - & - & - & - & - & This study \\
\hline
\end{tabular}

SS, secretary signal sequence; EGF, EGF-like domain; THR, the C terminal hydrophobic region. Ref: references. ZJ/HB and YN represented isolates from Zhejiang/ Hubei and Yunnan Provinces in China, respectively. • indicates identical amino acid residues compared to the Salvador I strain.

Zhejiang and Hubei isolates [32], but it was very common in South Asian isolates such as those from Bangladesh [25], Thailand [15], India [26], and also in Yunnan isolates.

Among a total of 24 amino acid substitutions in Pvs28 from worldwide isolates, M52L and T140S were the most common substitutions from different geographic regions [15,24-26,31]. The amino acid residues L98I and L116V were very common in South Asia isolates such as those from Bangladesh [25], Thailand [15], India [26], and also in Yunnan isolates. The amino acid residue T65K was not identified in isolates from Yunnan and South Korea [22], but was common in those from Bangladesh [25], Thailand [33], India [25,26] and Iran [24].

\section{Genetic diversity of pvs 25 and pvs 28}

There were a total of four polymorphic sites that generated five haplotypes in $p v s 25$. There were a total of 12 polymorphic sites that yielded eight haplotypes in $p v s 28$. Nucleotide diversity of $p v s 28(\pi=0.0034 \pm 0.0012)$ was significantly higher than that of pvs25 $(\pi=0.0013 \pm$ $0.0009)(p<0.05)$ (Table 5).

\section{Discussion}

In this study, we assessed the level of genetic diversity of pvs 25 and pvs 28 genes in P. vivax isolates from Yunnan Province, China. A total of four and eight amino acid substitutions have been identified in Pvs25 and Pvs28, respectively. Compared with $P$. vivax isolates from previous studies conducted in Asia, our results showed that the majority of the variant amino acids of Pvs25 and Pvs28 detected in Yunnan isolates were shared with those reported in isolates from South Asia (Bangladesh, Thailand and India) $[15,25]$. Because of its location in southwest China, Yunnan isolates had mutant residues characteristic of South Asian isolates, such as mutant residues E97Q and Q131K in Pvs25, as well as L98I and L116V in Pvs28. It suggested that some parasite haplotypes may have different geographic distributions.

One of the major obstacles to the development of an effective malaria vaccine is the genetic polymorphism of many of the genes in natural parasite populations that otherwise would be promising vaccine candidates. A number of studies have shown that TBVs candidates have limited polymorphism compared to antigens expressed in asexual stage parasites [34,35]. Our data showed that pvs25 $(\pi=0.0013)$ had a much lower level of diversity than $P$. vivax blood stage proteins DBP $(\pi=$ 0.0086-0.0184) [36] and MSP1 $(\pi=0.1193-0.2055)$ [37]. In addition, compared with $P$. falciparum sexual stage proteins pfs 25 ( $\pi=0.0035)$ [34], the lower genetic diversity of pvs 25 suggested that genetic polymorphism of pvs 25 was limited in Yunnan isolates. Although the nucleotide diversity of pvs 28 was significantly higher $(\pi$ $=0.0034$ ) than that of $p v s 25$, it was still conserved when compared with the blood stage vaccine candidates. 
Table 4 Amino acid variations of Pvs28 among worldwide isolates

\begin{tabular}{|c|c|c|c|c|c|c|c|c|c|c|c|c|c|c|c|c|c|c|c|c|c|c|c|c|c|c|}
\hline \multirow[b]{2}{*}{ Country } & \multicolumn{2}{|c|}{ SS } & \multicolumn{2}{|c|}{ EGF-1 } & \multicolumn{8}{|c|}{ EGF-2 } & \multicolumn{4}{|c|}{ EGF-3 } & $\frac{\text { EGF-4 }}{1}$ & & \multicolumn{4}{|c|}{ RR } & & \multicolumn{2}{|c|}{ THR } & \multirow[b]{2}{*}{ Ref. } \\
\hline & $\begin{array}{l}0 \\
0 \\
5\end{array}$ & $\begin{array}{l}0 \\
1 \\
4\end{array}$ & $\begin{array}{l}0 \\
5 \\
2\end{array}$ & $\begin{array}{l}0 \\
5 \\
3\end{array}$ & $\begin{array}{l}0 \\
6 \\
5\end{array}$ & $\begin{array}{l}0 \\
7 \\
9\end{array}$ & $\begin{array}{l}0 \\
8 \\
1\end{array}$ & $\begin{array}{l}0 \\
8 \\
7\end{array}$ & $\begin{array}{l}0 \\
9 \\
5\end{array}$ & $\begin{array}{l}0 \\
9 \\
8\end{array}$ & $\begin{array}{l}1 \\
0 \\
5\end{array}$ & $\begin{array}{l}1 \\
0 \\
6\end{array}$ & $\begin{array}{l}1 \\
1 \\
0\end{array}$ & $\begin{array}{l}1 \\
1 \\
3\end{array}$ & $\begin{array}{l}1 \\
1 \\
6\end{array}$ & $\begin{array}{l}1 \\
4 \\
0\end{array}$ & $\begin{array}{l}1 \\
5 \\
9\end{array}$ & $\begin{array}{l}1 \\
7 \\
9\end{array}$ & $\begin{array}{l}1 \\
9 \\
1\end{array}$ & $\begin{array}{l}2 \\
0 \\
8 \\
* \\
\end{array}$ & $\begin{array}{l}2 \\
1 \\
0 \\
\#\end{array}$ & $\begin{array}{l}2 \\
1 \\
4 \\
\$ \\
\end{array}$ & GSGGE/D & $\begin{array}{l}2 \\
2 \\
1 \\
\&\end{array}$ & $\begin{array}{l}2 \\
2 \\
2 \\
@\end{array}$ & \\
\hline Sal-I & $\mathrm{H}$ & V & M & A & $T$ & V & A & D & G & L & E & V & N & $\mathrm{N}$ & L & $T$ & K & G & G & D & G & S & 6 & V & I & AAC99770 \\
\hline Iran & . & $\cdot$ & $\mathrm{L}$ & . & $T / K$ & $\cdot$ & $\cdot$ & . & $\cdot$ & $\cdot$ & $\cdot$ & $\cdot$ & $\cdot$ & $\cdot$ & $\cdot$ & S & $\cdot$ & $\cdot$ & . & $\cdot$ & $\cdot$ & $\cdot$ & $4-6$ & $\cdot$ & $\cdot$ & [24] \\
\hline Mexico & $\cdot$ & $\cdot$ & L & $\cdot$ & $\cdot$ & $\cdot$ & $\cdot$ & N & $\cdot$ & $\cdot$ & $\cdot$ & $\cdot$ & Y & $\cdot$ & $\cdot$ & $S$ & $\cdot$ & $\cdot$ & $\cdot$ & $\cdot$ & $\cdot$ & $\cdot$ & $5-6$ & $\cdot$ & $\cdot$ & [31] \\
\hline Bangladesh & $\cdot$ & $\cdot$ & $M / L$ & $\cdot$ & $T / K$ & $\cdot$ & $\cdot$ & $\cdot$ & $\cdot$ & $\mathrm{L} / \mathrm{l}$ & $\cdot$ & $\cdot$ & $\cdot$ & N/S & $L N$ & $\mathrm{~T} / \mathrm{S}$ & $\begin{array}{l}\mathrm{K} \\
\mathrm{R}\end{array}$ & $\cdot$ & $\cdot$ & $\cdot$ & $\cdot$ & $\cdot$ & $5-7$ & $\cdot$ & $\begin{array}{l}\text { I/ } \\
M\end{array}$ & [25] \\
\hline Thailand & $\cdot$ & $\cdot$ & $M / L$ & AN & $T / K$ & $\cdot$ & AN & $\cdot$ & $\mathrm{G} / \mathrm{N}$ & L/I & $E / K$ & V/E & $\cdot$ & $\cdot$ & $L / N$ & $\mathrm{~T} / \mathrm{S}$ & $\cdot$ & $\cdot$ & $\cdot$ & $\cdot$ & $\cdot$ & $\cdot$ & $5-7$ & $\cdot$ & $\cdot$ & [15] \\
\hline India & $\cdot$ & . & $\mathrm{L}$ & $\cdot$ & K & $\cdot$ & $\cdot$ & . & $\cdot$ & $\cdot$ & $\cdot$ & $\cdot$ & . & . & $\cdot$ & $\mathrm{S}$ & . & . & . & · & $\cdot$ & . & 4 & $\cdot$ & $\cdot$ & [25] \\
\hline India & $\mathrm{H} / \mathrm{T} / \mathrm{Y}$ & $\cdot$ & $M / L$ & AN & $\mathrm{T} / \mathrm{K}$ & $\mathrm{V} / \mathrm{E}$ & $\cdot$ & $\cdot$ & $\cdot$ & L/I & $E / K$ & $\cdot$ & $\cdot$ & $\cdot$ & $L N$ & $\mathrm{~T} / \mathrm{S}$ & $\cdot$ & $\cdot$ & $\mathrm{D}$ & $\mathrm{D} / \mathrm{G}$ & $G / R$ & $\begin{array}{l}\mathrm{S} / \\
\mathrm{T}\end{array}$ & $3-6$ & $\mathrm{~V} / \mathrm{L}$ & I/M & [26] \\
\hline S Korea & $\cdot$ & $\cdot$ & $L$ & $\cdot$ & $\cdot$ & $\cdot$ & $\cdot$ & $\cdot$ & $\cdot$ & $\cdot$ & $\cdot$ & $\cdot$ & $\cdot$ & $\cdot$ & $\cdot$ & S & $\cdot$ & $\begin{array}{c}\text { G/ } \\
\text { E }\end{array}$ & $\begin{array}{l}\mathrm{G} / \\
\mathrm{R}\end{array}$ & $\cdot$ & $\cdot$ & $\cdot$ & 6 & $\cdot$ & $\cdot$ & [22] \\
\hline China (YN) & - & $V / L$ & $M / L$ & . & - & - & - & . & • & $L / I$ & $E / K$ & - & - & - & $L / N$ & $\mathrm{~T} / \mathrm{S}$ & - & $\mathrm{G} / \mathrm{E}$ & - & - & - & - & $5-6$ & - & I/M & This study \\
\hline
\end{tabular}

SS, secretary signal sequence; EGF, EGF-like domain; RR, repeat regions; THR, the C terminal hydrophobic region. Ref.: references. YN represented isolates from Yunnan Provinces in China. • indicates identical amino acid residues compared to the Salvador I strain.

*, \#, \$, \&, @ represented amino acid residues 210, 212, 216, 223, 224 reported in reference 26, respectively. 
Table 5 Nucleotide diversity of the pvs25 and pvs28 genes

\begin{tabular}{lllllc}
\hline Gene & $\mathbf{n}$ & $\boldsymbol{S}$ & $\boldsymbol{h}$ & $\mathbf{H d}$ & $\boldsymbol{\pi} \pm \mathbf{S E}$ \\
\hline pvs25 & 30 & 4 & 5 & 0.671 & $0.0013 \pm 0.0009$ \\
pvs28 & 30 & 12 & 8 & 0.556 & $0.0034 \pm 0.0012$ \\
\hline
\end{tabular}

$\mathrm{n}$, number of sequences samples; $S$, number of polymorphic sites; $h$, number of haplotypes; $\mathrm{Hd}$, haplotype diversity; $\pi$, nucleotide diversity; nucleotide diversity with its standard error (SE) were computed with Jukes-Cantor correction using MEGA 4.0.

Although direct sequencing of the PCR products in our study might underestimate the genetic diversity of these two genes, the effect should be minor since our study areas are malaria hypoendemic and mixed infections should not be prevalent. The limited diversity of sexual stage antigens such as P25 and P28 is perhaps attributed to the expression of these proteins in mosquito stages, which should avoid immune selection in the humans.

It is very important to evaluate the effect of genetic polymorphism of Pvs25 and Pvs28 on the efficacy of Sal-I based TBVs in different malaria epidemiological areas. The transmission-blocking assays conducted in Thailand clearly demonstrated that antisera to recombinant Pvs25 and Pvs28 based on the Sal-I strain of $P$. vivax recognized corresponding molecules expressed by field-isolated parasites in Thailand. Our data showed limited genetic diversity of pvs 25 and pvs 28 in the Yunnan isolates. The major amino acid haplotype of Pvs25 from this region was also shared with that from Thailand isolates, it provids a good prospect on implication of TBVs in China malaria areas.

Altogether, our study offers a first glimpse of the genetic diversity of two TBVs candidate antigens in Yunnan Province, China. Yet, this study only offered limited information of $P$. vivax parasites from Yunnan. Further studies on a larger sample scale and comparison analysis with Central China isolates will certainly improve our understanding of genetic polymorphisms of TBVs candidates in the malaria endemic areas of China.

\section{Conclusions}

TBVs are one of the important strategies for controlling malaria transmission. A key point in vaccine optimization is to understand the extent of genetic diversity of candidate antigens. The TBVs candidates Pvs25 and Pvs28 from Yunnan isolates showed limited genetic diversity. Further studies encompassing larger malaria endemic areas are needed to provide a thorough evaluation of situation in China.

\section{Acknowledgements and Funding}

We are grateful to Drs. Osamu Kaneko, Takafumi Tsuboi and Motomi Torii for the helpful suggestions. The work was supported by the National S \& T Major Program (2008ZX10004-011), National Natural Science Foundation of China (30972774) and National Institutes of Health (U19AI089672).

\begin{abstract}
Author details
${ }^{1}$ Department of Immunology, College of Basic Medical Sciences, China Medical University, Shenyang, China. ${ }^{2}$ Institute of Pathology and Pathophysiology, China Medical University, Shenyang, China. ${ }^{3}$ Department of Parasitology, College of Basic Medical Sciences, Dali Medical College, Dali, China. ${ }^{4}$ Department of Microbiology and Parasitology, Institute of Basic Medical Sciences, Chinese Academy of Medical Sciences \& School of Basic Medicine, Peking Union Medical College, Beijing, China. ${ }^{5}$ Department of Entomology, Pennsylvania State University, University Park, Pennsylvania, USA.
\end{abstract}

\section{Authors' contributions}

YC conceived of the study and helped to draft the manuscript. YY collected the blood spots specimens. HF carried out the studies, statistical analysis and drafted the manuscript. $L Z, X Z, G W, Y P, Y L, Y L$ participated in the molecular genetic studies and sequence alignment. LC helped with statistical analysis and critically revised the manuscript. All authors contributed to the writing of the manuscript and approved the submitted version of the manuscript.

\section{Competing interests}

The authors declare that they have no competing interests.

Received: 20 April 2011 Accepted: 28 November 2011

Published: 28 November 2011

\section{References}

1. World Health Organization: World malaria report 2009. Geneva 2009.

2. Mendis K, Sina BJ, Marchesini P, Carter R: The neglected burden of Plasmodium vivax malaria. Am J Trop Med Hyg 2001, 64:97-106.

3. Zhou SS, Wang Y, Xia ZG: Malaria situation in the People's Republic Of China in 2009. Zhongguo Ji Sheng Chong Xue Yu Ji Sheng Chong Bing Za Zhi 2011, 29:1-3.

4. Ministry of Health of the People's Republic of China: China to eliminate Malaria Action Plan (2010-2020). 2010.

5. Cui L, Yan G, Sattabongkot J, Cao Y, Chen B, Chen X, Fan Q, Fang Q, Jongwutiwes S, Parker D, Sirichaisinthop J, Kyaw MP, Su XZ, Yang H, Yang Z, Wang B, Xu J, Zheng B, Zhong D, Zhou G: Malaria in the Greater Mekong Subregion: Heterogeneity and complexity. Acta Trop 2011.

6. Clements AC, Barnett AG, Cheng ZW, Snow RW, Zhou HN: Space-time variation of malaria incidence in Yunnan province, China. Malar J 2009, 8:180.

7. Zhou SS, Wang Y, Tang LH: [Malaria situation in the People's Republic of China in 2006]. Zhongguo Ji Sheng Chong Xue Yu Ji Sheng Chong Bing Za Zhi 2007, 25:439-441.

8. Zhou SS, Wang Y, Fang W, Tang LH: [Malaria situation in the People's Republic Of China in 2007]. Zhongguo Ji Sheng Chong Xue Yu Ji Sheng Chong Bing Za Zhi 2008, 26:401-403.

9. Zhou SS, Wang Y, Fang W, Tang LH: [Malaria situation in the People's Republic of China in 2008]. Zhongguo Ji Sheng Chong Xue Yu Ji Sheng Chong Bing Za Zhi 2009, 27:457, 455-456.

10. Lin H, Lu L, Tian L, Zhou S, Wu H, Bi Y, Ho SC, Liu Q: Spatial and temporal distribution of falciparum malaria in China. Malar J 2009, 8:130.

11. Kaslow DC: Transmission blocking vaccines. In Malaria Vaccine Development. Edited by: Hofman SL. ASM books; 1996:181-228.

12. Tsuboi T, Tachibana M, Kaneko O, Torii M: Transmission-blocking vaccine of vivax malaria. Parasitol Int 2003, 52:1-11.

13. Tomas AM, Margos G, Dimopoulos G, van Lin LH, de Koning-Ward TF, Sinha R, Lupetti P, Beetsma AL, Rodriguez MC, Karras M, Hager A, Mendoza J, Butcher GA, Kafatos F, Janse CJ, Waters AP, Sinden RE: P25 and P28 proteins of the malaria ookinete surface have multiple and partially redundant functions. EMBO J 2001, 20:3975-3983.

14. Moon SU, Kim HH, Kim TS, Choi KM, Oh CM, Ahn YJ, Hwang SK, Sohn Y, Shin EH, Kim H, Lee HW: Blocking effect of a monoclonal antibody against recombinant Pvs25 on sporozoite development in Anopheles sinensis. Clin Vaccine Immunol 2010, 17:1183-1187.

15. Sattabongkot J, Tsuboi T, Hisaeda H, Tachibana M, Suwanabun N, Rungruang T, Cao YM, Stowers AW, Sirichaisinthop J, Coleman RE, Torii M: Blocking of transmission to mosquitoes by antibody to Plasmodium vivax malaria vaccine candidates Pvs25 and Pvs28 despite antigenic polymorphism in field isolates. Am J Trop Med Hyg 2003, 69:536-541. 
16. Miyata T, Harakuni T, Tsuboi T, Sattabongkot J, Kohama H, Tachibana M, Matsuzaki G, Torii M, Arakawa T: Plasmodium vivax ookinete surface protein Pvs25 linked to cholera toxin B subunit induces potent transmission-blocking immunity by intranasal as well as subcutaneous immunization. Infect Immun 2010, 78:3773-3782.

17. Blagborough AM, Yoshida S, Sattabongkot J, Tsuboi T, Sinden RE: Intranasal and intramuscular immunization with Baculovirus Dual Expression System-based Pvs25 vaccine substantially blocks Plasmodium vivax transmission. Vaccine 2010, 28:6014-6020.

18. Saul A, Hensmann M, Sattabongkot J, Collins WE, Barnwell JW, Langermans JA, Wu Y, Long CA, Dubovsky F, Thomas AW: Immunogenicity in rhesus of the Plasmodium vivax mosquito stage antigen Pvs25H with Alhydrogel and Montanide ISA 720. Parasite Immunol 2007, 29:525-533.

19. Malkin EM, Durbin AP, Diemert DJ, Sattabongkot J, Wu Y, Miura K, Long CA, Lambert L, Miles AP, Wang J, Stowers A, Miller LH, Saul A: Phase 1 vaccine trial of Pvs25H: a transmission blocking vaccine for Plasmodium vivax malaria. Vaccine 2005, 23:3131-3138.

20. Wu Y, Ellis RD, Shaffer D, Fontes E, Malkin EM, Mahanty S, Fay MP, Narum D, Rausch K, Miles AP, Aebig J, Orcutt A, Muratova O, Song G, Lambert L, Zhu D, Miura K, Long C, Saul A, Miller LH, Durbin AP: Phase 1 trial of malaria transmission blocking vaccine candidates Pfs 25 and Pvs25 formulated with montanide ISA 51. PLoS One 2008, 3:e2636.

21. Tsuboi T, Kaslow DC, Gozar MM, Tachibana M, Cao YM, Torii M: Sequence polymorphism in two novel Plasmodium vivax ookinete surface proteins, Pvs25 and Pvs28, that are malaria transmission-blocking vaccine candidates. Mol Med 1998, 4:772-782.

22. Han ET, Lee WJ, Sattabongkot J, Jang JW, Nam MH, An SS, Suh I, Lim CS: Sequence polymorphisms of Plasmodium vivax ookinete surface proteins (Pvs25 and Pvs28) from clinical isolates in Korea. Trop Med Int Health 2010, 15:1072-1076.

23. Escalante AA, Cornejo OE, Freeland DE, Poe AC, Durrego E, Collins WE, Lal AA: A monkey's tale: the origin of Plasmodium vivax as a human malaria parasite. Proc Natl Acad Sci USA 2005, 102:1980-1985.

24. Zakeri S, Razavi S, Djadid ND: Genetic diversity of transmission blocking vaccine candidate (Pvs25 and Pvs28) antigen in Plasmodium vivax clinical isolates from Iran. Acta Trop 2009, 109:176-180.

25. Tsuboi T, Kaneko O, Cao YM, Tachibana M, Yoshihiro Y, Nagao T, Kanbara H, Torii M: A rapid genotyping method for the vivax malaria transmissionblocking vaccine candidates, Pvs25 and Pvs28. Parasitol Int 2004, 53:211-216.

26. Prajapati SK, Joshi H, Dua VK: Antigenic repertoire of Plasmodium vivax transmission-blocking vaccine candidates from the Indian subcontinent. Malar J 2011, 10:111.

27. Hui FM, Xu B, Chen ZW, Cheng X, Liang L, Huang HB, Fang LQ, Yang H, Zhou HN, Yang HL, Zhou XN, Cao WC, Gong P: Spatio-temporal distribution of malaria in Yunnan Province, China. Am J Trop Med Hyg 2009, 81:503-509.

28. Cox FE: History of the discovery of the malaria parasites and their vectors. Parasit Vectors 2010, 3:5.

29. Thompson JD, Higgins DG, Gibson TJ: CLUSTAL W: improving the sensitivity of progressive multiple sequence alignment through sequence weighting, position-specific gap penalties and weight matrix choice. Nucleic Acids Res 1994, 22:4673-4680.

30. Rozas J, Sanchez-DelBarrio JC, Messeguer X, Rozas R: DnaSP, DNA polymorphism analyses by the coalescent and other methods. Bioinformatics 2003, 19:2496-2497.

31. Gonzalez-Ceron L, Alvarado-Delgado A, Martinez-Barnetche J, Rodriguez MH, Ovilla-Munoz M, Perez F, Hernandez-Avila JE, Sandoval MA, Rodriguez Mdel C, Villarreal-Trevino C: Sequence variation of ookinete surface proteins Pvs25 and Pvs28 of Plasmodium vivax isolates from Southern Mexico and their association to local anophelines infectivity. Infect Genet Evol 2010, 10:645-654.

32. Zheng $L, X u$ WM, Liu YJ, Yang YM, Cao YM: Transmission-blocking vaccine candidate of Plasmodium vivax Pvs25 is highly conservative among Chinese isolates. Zhongguo Ji Sheng Chong Xue Yu Ji Sheng Chong Bing Za Zhi 2004, 22:16-19.

33. Sattabongkot J, Tsuboi T, Zollner GE, Sirichaisinthop J, Cui L: Plasmodium vivax transmission: chances for control? Trends Parasitol 2004, 20:192-198.

34. Escalante AA, Lal AA, Ayala FJ: Genetic polymorphism and natural selection in the malaria parasite Plasmodium falciparum. Genetics 1998, 149:189-202.
35. Barry AE, Schultz L, Buckee CO, Reeder JC: Contrasting population structures of the genes encoding ten leading vaccine-candidate antigens of the human malaria parasite, Plasmodium falciparum. PLoS One 2009, 4:e8497.

36. Cole-Tobian J, King CL: Diversity and natural selection in Plasmodium vivax Duffy binding protein gene. Mol Biochem Parasitol 2003, 127:121-132

37. Putaporntip C, Jongwutiwes S, Sakihama N, Ferreira MU, Kho WG, Kaneko A, Kanbara H, Hattori T, Tanabe K: Mosaic organization and heterogeneity in frequency of allelic recombination of the Plasmodium vivax merozoite surface protein-1 locus. Proc Natl Acad Sci USA 2002, 99:16348-16353.

doi:10.1186/1756-3305-4-224

Cite this article as: Feng et al:: Genetic diversity of transmissionblocking vaccine candidates Pvs25 and Pvs28 in Plasmodium vivax isolates from Yunnan Province, China. Parasites \& Vectors 2011 4:224.

\section{Submit your next manuscript to BioMed Central and take full advantage of:}

- Convenient online submission

- Thorough peer review

- No space constraints or color figure charges

- Immediate publication on acceptance

- Inclusion in PubMed, CAS, Scopus and Google Scholar

- Research which is freely available for redistribution 